\title{
Job frosty-rain-49a3
}

This output file was generated at:

2019-Mar-18 06:39:58 PDT

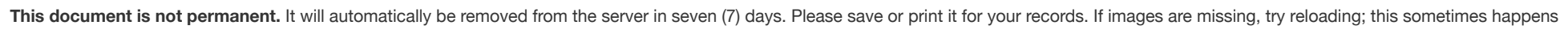
under heavy server load.

Single file archive including this page, all images, all clumpp files: download. [.tar.gz]

\section{L(K)}

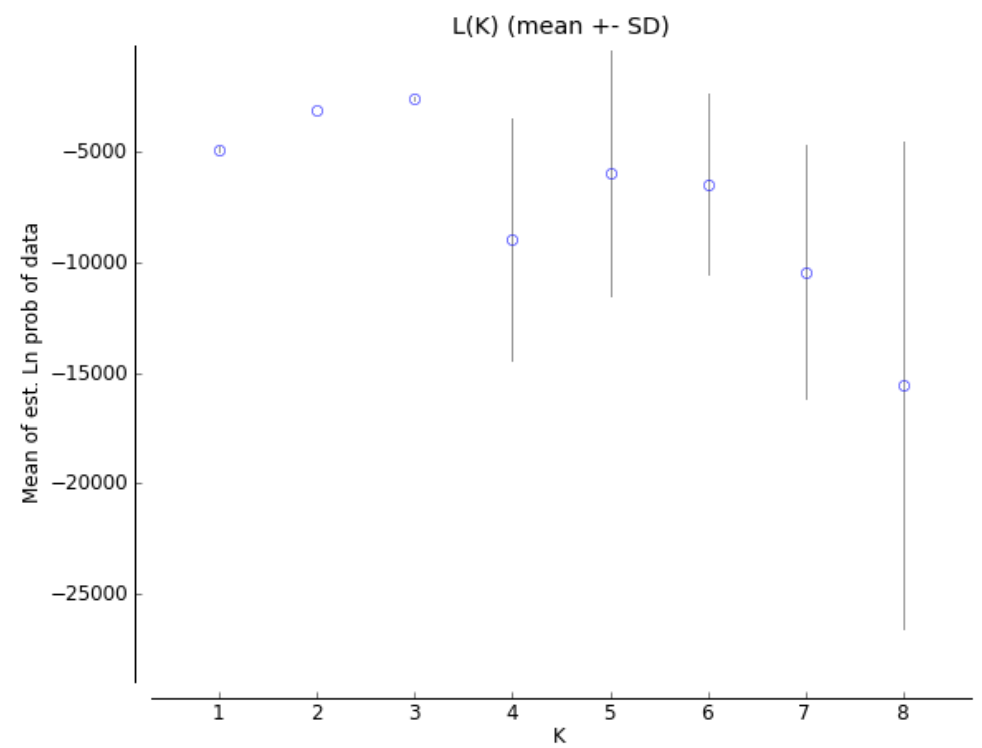

$\mathrm{L}(\mathrm{K}): \mathrm{pdf}$ eps

\section{Clumpp files}

\section{$\mathrm{K}=1$ Clumpp indfile}

$\mathrm{K}=2$ Clumpp indfile

$\mathrm{K}=3$ Clumpp indfile

$\mathrm{K}=4$ Clumpp indfile

$\mathrm{K}=5$ Clumpp indfile

$\mathrm{K}=6$ Clumpp indfile

$\mathrm{K}=7$ Clumpp indfile

$\mathrm{K}=8$ Clumpp indfile

\section{Evanno method}

"Evanno et al., 2005. Molecular Ecology 14, 2611 - 2620. How are we calculating this? Look at the FAQ. 


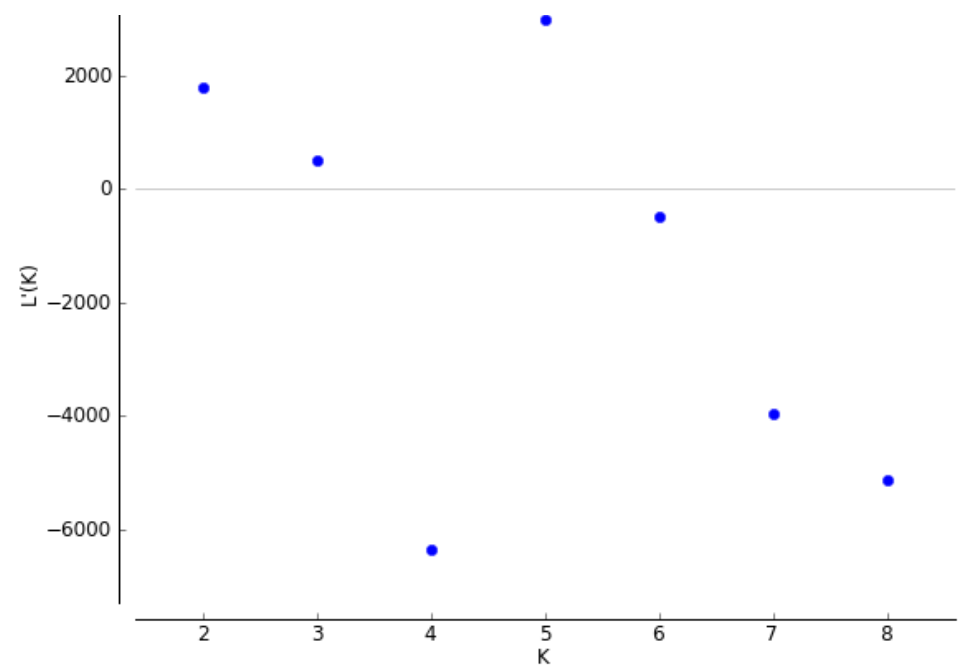

$L^{\prime}(K):$ pdf eps

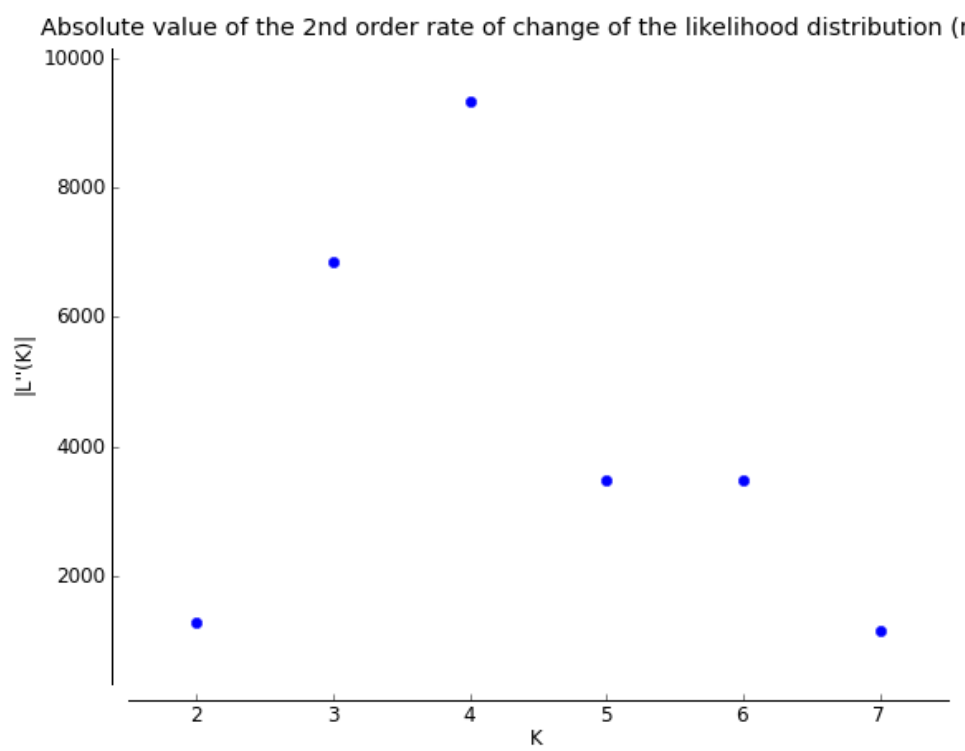

$\left|L^{\prime \prime}(K)\right|:$ pdf eps

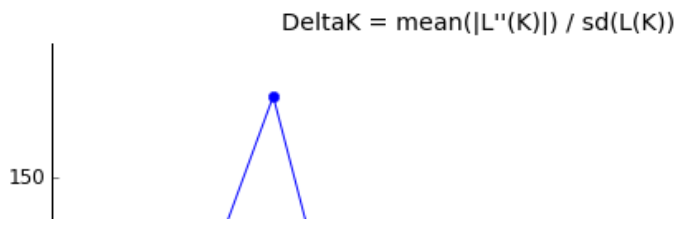




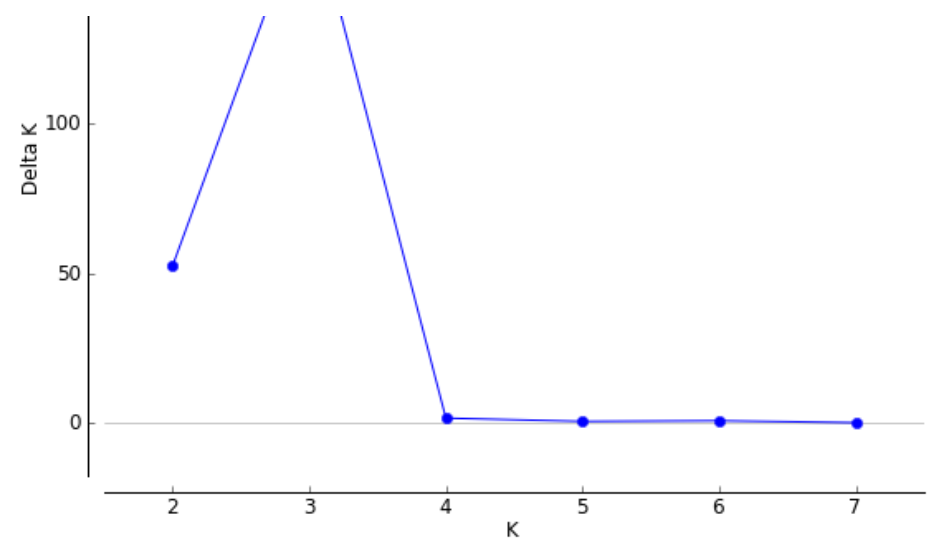

Delta K: pdf eps

The Evanno table output is also available as a tab-delimited text file (for use with Excel) here.

$\begin{array}{ccccccc}\mathbf{K} & \text { Reps } & \text { Mean } \operatorname{LnP(K)} & \text { Stdev LnP(K) } & \operatorname{Ln}^{\prime}(\mathbf{K}) & \left|\mathbf{L n}^{\prime \prime}(\mathbf{K})\right| & \text { Delta K } \\ 1 & 10 & -4917.800000 & 106.862050 & - & - & - \\ 2 & 10 & -3129.340000 & 24.239509 & 1788.460000 & 1273.760000 & 52.548920 \\ 3 & 10 & -2614.640000 & 38.807078 & 514.700000 & 6864.540000 & 176.888864 \\ 4 & 10 & -8964.480000 & 5467.302233 & -6349.840000 & 9340.680000 & 1.708462 \\ 5 & 10 & -5973.640000 & 5517.389051 & 2990.840000 & 3485.430000 & 0.631717 \\ 6 & 10 & -6468.230000 & 4100.908499 & -494.590000 & 3468.900000 & 0.845886 \\ 7 & 10 & -10431.720000 & 5748.485227 & -3963.490000 & 1148.408182 & 0.199776 \\ 8 & 11 & -15543.618182 & 11008.014977 & -5111.898182 & - & -\end{array}$

\section{Raw STRUCTURE output}

The raw STRUCTURE output is also available as a tab-delimited text file (for use with Excel) here.

\begin{tabular}{|c|c|c|c|c|c|}
\hline File name & $\begin{array}{c}\text { Run } \\
\#\end{array}$ & K & $\begin{array}{l}\text { Est. Ln prob. of } \\
\text { data }\end{array}$ & $\begin{array}{l}\text { Mean value of } \mathrm{Ln} \\
\text { likelihood }\end{array}$ & $\begin{array}{l}\text { Variance of Lr } \\
\text { likelihood }\end{array}$ \\
\hline $\begin{array}{l}\text { Results1000- } \\
10000 \_r u n \_6 \_f\end{array}$ & 6 & 1 & -4810.8 & -4392.8 & 836.0 \\
\hline $\begin{array}{l}\text { Results1000- } \\
\text { 10000_run_9_f }\end{array}$ & 9 & 1 & -4888.2 & -4396.6 & 983.2 \\
\hline $\begin{array}{l}\text { Results1000- } \\
\text { 10000_run_10_f }\end{array}$ & 10 & 1 & -4813.7 & -4392.1 & 843.2 \\
\hline $\begin{array}{l}\text { Results1000- } \\
\text { 10000_run_11_f }\end{array}$ & 11 & 1 & -5045.1 & -4411.4 & 1267.3 \\
\hline $\begin{array}{l}\text { Results1000- } \\
\text { 10000_run_8_f }\end{array}$ & 8 & 1 & -4873.2 & -4395.7 & 955.1 \\
\hline $\begin{array}{l}\text { Results1000- } \\
\text { 10000_run_7_f }\end{array}$ & 7 & 1 & -4992.6 & -4404.3 & 1176.7 \\
\hline $\begin{array}{l}\text { Results1000- } \\
\text { 10000_run_5_f }\end{array}$ & 5 & 1 & -5038.9 & -4418.1 & 1241.6 \\
\hline $\begin{array}{l}\text { Results1000- } \\
\text { 10000_run_2_f }\end{array}$ & 2 & 1 & -4826.3 & -4393.0 & 866.5 \\
\hline $\begin{array}{l}\text { Results1000- } \\
\text { 10000_run_4_f }\end{array}$ & 4 & 1 & -4819.4 & -4392.6 & 853.7 \\
\hline $\begin{array}{l}\text { Results1000- } \\
\text { 10000_run_3_f }\end{array}$ & 3 & 1 & -5069.8 & -4412.2 & 1315.2 \\
\hline $\begin{array}{l}\text { Results1000- } \\
\text { 10000_run_13_f }\end{array}$ & 13 & 2 & -3118.6 & -2731.2 & 774.8 \\
\hline $\begin{array}{l}\text { Results1000- } \\
\text { 10000_run_18_f }\end{array}$ & 18 & 2 & -3076.7 & -2731.0 & 691.5 \\
\hline $\begin{array}{l}\text { Results1000- } \\
\text { 10000_run_14_f }\end{array}$ & 14 & 2 & -3131.1 & -2734.3 & 793.6 \\
\hline $\begin{array}{l}\text { Results1000- } \\
\text { 10000_run_16_f }\end{array}$ & 16 & 2 & -3132.3 & -2726.9 & 810.8 \\
\hline $\begin{array}{l}\text { Results1000- } \\
\text { 10000_run_15_f }\end{array}$ & 15 & 2 & -3150.6 & -2740.5 & 820.1 \\
\hline $\begin{array}{l}\text { Results1000- } \\
\text { 10000_run_12_f }\end{array}$ & 12 & 2 & -3133.3 & -2739.0 & 788.6 \\
\hline $\begin{array}{l}\text { Results1000- } \\
\text { 10000_run_19_f }\end{array}$ & 19 & 2 & -3143.7 & -2736.2 & 815.1 \\
\hline $\begin{array}{l}\text { Results1000- } \\
\text { 10000_run_17_f }\end{array}$ & 17 & 2 & -3132.5 & -2732.9 & 799.2 \\
\hline Results1000- & 20 & 2 & -3165.4 & -2754.8 & 821.3 \\
\hline
\end{tabular}




\begin{tabular}{|c|c|c|c|c|c|}
\hline 10000_run_20_f & -- & - & . & $\cdots$ & $-\cdots$ \\
\hline $\begin{array}{l}\text { Results1000- } \\
\text { 10000_run_21_f }\end{array}$ & 21 & 2 & -3109.2 & -2730.3 & 757.8 \\
\hline $\begin{array}{l}\text { Results1000- } \\
\text { 10000_run_25_f }\end{array}$ & 25 & 3 & -2632.2 & -2173.5 & 917.6 \\
\hline $\begin{array}{l}\text { Results1000- } \\
\text { 10000_run_27_f }\end{array}$ & 27 & 3 & -2620.3 & -2162.1 & 916.5 \\
\hline $\begin{array}{l}\text { Results1000- } \\
\text { 10000_run_23_f }\end{array}$ & 23 & 3 & -2615.6 & -2172.5 & 886.2 \\
\hline $\begin{array}{l}\text { Results1000- } \\
\text { 10000_run_29_f }\end{array}$ & 29 & 3 & -2696.6 & -2170.4 & 1052.4 \\
\hline $\begin{array}{l}\text { Results1000- } \\
\text { 10000_run_24_f }\end{array}$ & 24 & 3 & -2548.5 & -2168.1 & 760.9 \\
\hline $\begin{array}{l}\text { Results1000- } \\
\text { 10000_run_22_f }\end{array}$ & 22 & 3 & -2600.3 & -2160.6 & 879.4 \\
\hline $\begin{array}{l}\text { Results1000- } \\
\text { 10000_run_26_f }\end{array}$ & 26 & 3 & -2588.6 & -2169.5 & 838.1 \\
\hline $\begin{array}{l}\text { Results } 1000- \\
\text { 10000_run_30_f }\end{array}$ & 30 & 3 & -2587.5 & -2167.2 & 840.7 \\
\hline $\begin{array}{l}\text { Results1000- } \\
\text { 10000_run_31_f }\end{array}$ & 31 & 3 & -2636.2 & -2165.3 & 941.8 \\
\hline $\begin{array}{l}\text { Results1000- } \\
\text { 10000_run_28_f }\end{array}$ & 28 & 3 & -2620.6 & -2159.4 & 922.5 \\
\hline $\begin{array}{l}\text { Results1000- } \\
\text { 10000_run_41_f }\end{array}$ & 41 & 4 & -17605.1 & -2033.0 & 31144.3 \\
\hline $\begin{array}{l}\text { Results1000- } \\
\text { 10000_run_36_f }\end{array}$ & 36 & 4 & -14379.6 & -1788.2 & 25182.9 \\
\hline $\begin{array}{l}\text { Results1000- } \\
\text { 10000_run_35_f }\end{array}$ & 35 & 4 & -2337.1 & -1720.4 & 1233.4 \\
\hline $\begin{array}{l}\text { Results1000- } \\
\text { 10000_run_39_f }\end{array}$ & 39 & 4 & -14555.5 & -1895.9 & 25319.1 \\
\hline $\begin{array}{l}\text { Results1000- } \\
\text { 10000_run_37_f }\end{array}$ & 37 & 4 & -8257.4 & -1877.0 & 12760.7 \\
\hline $\begin{array}{l}\text { Results1000- } \\
\text { 10000_run_40_f }\end{array}$ & 40 & 4 & -3255.6 & -2069.6 & 2371.9 \\
\hline $\begin{array}{l}\text { Results1000- } \\
\text { 10000_run_33_f }\end{array}$ & 33 & 4 & -4835.6 & -1810.9 & 6049.4 \\
\hline $\begin{array}{l}\text { Results1000- } \\
\text { 10000_run_38_f }\end{array}$ & 38 & 4 & -6315.1 & -1830.7 & 8968.7 \\
\hline $\begin{array}{l}\text { Results1000- } \\
\text { 10000_run_34_f }\end{array}$ & 34 & 4 & -5009.4 & -1764.7 & 6489.4 \\
\hline $\begin{array}{l}\text { Results1000- } \\
\text { 10000_run_32_f }\end{array}$ & 32 & 4 & -13094.4 & -1800.0 & 22588.8 \\
\hline $\begin{array}{l}\text { Results1000- } \\
\text { 10000_run_51_f }\end{array}$ & 51 & 5 & -3587.5 & -2096.0 & 2983.1 \\
\hline $\begin{array}{l}\text { Results } 1000- \\
\text { 10000_run_42_f }\end{array}$ & 42 & 5 & -3355.9 & -1746.3 & 3219.3 \\
\hline $\begin{array}{l}\text { Results1000- } \\
\text { 10000_run_50_f }\end{array}$ & 50 & 5 & -2551.8 & -1701.0 & 1701.5 \\
\hline $\begin{array}{l}\text { Results1000- } \\
\text { 10000_run_47_f }\end{array}$ & 47 & 5 & -3406.3 & -1684.2 & 3444.2 \\
\hline $\begin{array}{l}\text { Results1000- } \\
\text { 10000_run_48_f }\end{array}$ & 48 & 5 & -13496.7 & -1843.6 & 23306.3 \\
\hline $\begin{array}{l}\text { Results } 1000- \\
\text { 10000_run_45_f }\end{array}$ & 45 & 5 & -2372.0 & -1711.5 & 1321.0 \\
\hline $\begin{array}{l}\text { Results1000- } \\
\text { 10000_run_49_f }\end{array}$ & 49 & 5 & -4320.2 & -1687.5 & 5265.5 \\
\hline $\begin{array}{l}\text { Results1000- } \\
\text { 10000_run_44_f }\end{array}$ & 44 & 5 & -18697.1 & -1740.0 & 33914.2 \\
\hline $\begin{array}{l}\text { Results } 1000- \\
\text { 10000_run_46_f }\end{array}$ & 46 & 5 & -3286.8 & -1596.9 & 3379.9 \\
\hline $\begin{array}{l}\text { Results1000- } \\
\text { 10000_run_43_f }\end{array}$ & 43 & 5 & -4662.1 & -1732.0 & 5860.3 \\
\hline $\begin{array}{l}\text { Results1000- } \\
\text { 10000_run_52_f }\end{array}$ & 52 & 6 & -2666.9 & -1723.0 & 1887.9 \\
\hline $\begin{array}{l}\text { Results1000- } \\
\text { 10000_run_55_f }\end{array}$ & 55 & 6 & -2602.6 & -1714.0 & 1777.2 \\
\hline $\begin{array}{l}\text { Results1000- } \\
\text { 10000_run_59_f }\end{array}$ & 59 & 6 & -9087.8 & -1798.6 & 14578.4 \\
\hline $\begin{array}{l}\text { Results1000- } \\
\text { 10000_run_56_f }\end{array}$ & 56 & 6 & -11455.4 & -1881.5 & 19147.9 \\
\hline $\begin{array}{l}\text { Results1000- } \\
\text { 10000_run_54_f }\end{array}$ & 54 & 6 & -2387.7 & -1589.6 & 1596.2 \\
\hline
\end{tabular}




\begin{tabular}{|c|c|c|c|c|c|}
\hline 10000_run_61_f & 61 & 6 & -8679.1 & -1700.6 & 13957.1 \\
\hline $\begin{array}{l}\text { Results1000- } \\
\text { 10000_run_60_f }\end{array}$ & 60 & 6 & -3017.1 & -1735.6 & 2563.0 \\
\hline $\begin{array}{l}\text { Results1000- } \\
\text { 10000_run_58_f }\end{array}$ & 58 & 6 & -6033.8 & -1619.4 & 8828.7 \\
\hline $\begin{array}{l}\text { Results1000- } \\
\text { 10000_run_57_f }\end{array}$ & 57 & 6 & -4921.6 & -1593.7 & 6655.8 \\
\hline $\begin{array}{l}\text { Results1000- } \\
\text { 10000_run_53_f }\end{array}$ & 53 & 6 & -13830.3 & -1727.7 & 24205.2 \\
\hline $\begin{array}{l}\text { Results1000- } \\
\text { 10000_run_67_f }\end{array}$ & 67 & 7 & -18082.3 & -1692.1 & 32780.5 \\
\hline $\begin{array}{l}\text { Results1000- } \\
\text { 10000_run_65_f }\end{array}$ & 65 & 7 & -8748.8 & -1713.3 & 14071.0 \\
\hline $\begin{array}{l}\text { Results1000- } \\
\text { 10000_run_66_f }\end{array}$ & 66 & 7 & -6510.3 & -1642.9 & 9735.0 \\
\hline $\begin{array}{l}\text { Results1000- } \\
\text { 10000_run_68_f }\end{array}$ & 68 & 7 & -6598.0 & -1668.0 & 9860.0 \\
\hline $\begin{array}{l}\text { Results1000- } \\
\text { 10000_run_62_f }\end{array}$ & 62 & 7 & -16141.3 & -1746.2 & 28790.2 \\
\hline $\begin{array}{l}\text { Results1000- } \\
\text { 10000_run_71_f }\end{array}$ & 71 & 7 & -11176.3 & -1807.3 & 18738.1 \\
\hline $\begin{array}{l}\text { Results1000- } \\
\text { 10000_run_63_f }\end{array}$ & 63 & 7 & -7533.4 & -1796.1 & 11474.5 \\
\hline $\begin{array}{l}\text { Results1000- } \\
\text { 10000_run_64_f }\end{array}$ & 64 & 7 & -5899.0 & -1710.8 & 8376.5 \\
\hline $\begin{array}{l}\text { Results1000- } \\
\text { 10000_run_70_f }\end{array}$ & 70 & 7 & -20181.9 & -1879.8 & 36604.1 \\
\hline $\begin{array}{l}\text { Results1000- } \\
\text { 10000_run_69_f }\end{array}$ & 69 & 7 & -3445.9 & -1613.8 & 3664.2 \\
\hline $\begin{array}{l}\text { Results1000- } \\
\text { 10000_run_78_f }\end{array}$ & 78 & 8 & -23531.1 & -1876.2 & 43309.8 \\
\hline $\begin{array}{l}\text { Results1000- } \\
\text { 10000_run_79_f }\end{array}$ & 79 & 8 & -15149.5 & -1772.6 & 26753.8 \\
\hline $\begin{array}{l}\text { Results1000- } \\
\text { 10000_run_1_f }\end{array}$ & 1 & 8 & -2214.4 & -1598.5 & 1231.8 \\
\hline $\begin{array}{l}\text { Results1000- } \\
\text { 10000_run_77_f }\end{array}$ & 77 & 8 & -30754.6 & -1790.9 & 57927.4 \\
\hline $\begin{array}{l}\text { Results1000- } \\
\text { 10000_run_75_f }\end{array}$ & 75 & 8 & -25846.8 & -1778.9 & 48135.8 \\
\hline $\begin{array}{l}\text { Results1000- } \\
\text { 10000_run_80_f }\end{array}$ & 80 & 8 & -22541.1 & -1799.9 & 41482.4 \\
\hline $\begin{array}{l}\text { Results1000- } \\
\text { 10000_run_73_f }\end{array}$ & 73 & 8 & -8072.1 & -1632.3 & 12879.5 \\
\hline $\begin{array}{l}\text { Results1000- } \\
\text { 10000_run_74_f }\end{array}$ & 74 & 8 & -3777.3 & -1621.9 & 4310.7 \\
\hline $\begin{array}{l}\text { Results1000- } \\
\text { 10000_run_76_f }\end{array}$ & 76 & 8 & -8369.8 & -1533.7 & 13672.1 \\
\hline $\begin{array}{l}\text { Results } 1000- \\
\text { 10000_run_72_f }\end{array}$ & 72 & 8 & -28358.6 & -1596.6 & 53523.9 \\
\hline $\begin{array}{l}\text { Results1000- } \\
\text { 10000_run_81_f }\end{array}$ & 81 & 8 & -2364.5 & -1589.1 & 1550.8 \\
\hline
\end{tabular}

\section{CITATION}

Earl, Dent A. and vonHoldt, Bridgett M. (2012)

STRUCTURE HARVESTER: a website and program for visualizing

STRUCTURE output and implementing the Evanno method.

Conservation Genetics Resources vol. 4 (2) pp. 359-361 doi: 10.1007/s12686-011-9548-7

Core version: vA.2 July 2014

Plot version: vA. 1 November 2012

Web version: v0.6.94 July 2014 